\title{
Isolation and Characterization of Salmonella Jumbo-Phage pSal-SNUABM-04
}

\author{
Jun Kwon, Sang Guen Kim, Hyoun Joong Kim, Sib Sankar Giri $\mathbb{1}^{\circ}$, Sang Wha Kim $\mathbb{1}$, Sung Bin Lee \\ and Se Chang Park *(D)
}

Citation: Kwon, J.; Kim, S.G.; Kim, H.J.; Giri, S.S.; Kim, S.W.; Lee, S.B.; Park, S.C. Isolation and Characterization of Salmonella Jumbo-Phage pSalSNUABM-04. Viruses 2021, 13, 27. https: / / dx.doi.org/10.3390/v13010027

Academic Editor: Dann Turner Received: 11 November 2020 Accepted: 24 December 2020 Published: 25 December 2020

Publisher's Note: MDPI stays neutral with regard to jurisdictional claims in published maps and institutional affiliations.

Copyright: () 2020 by the authors. Licensee MDPI, Basel, Switzerland. This article is an open access article distributed under the terms and conditions of the Creative Commons Attribution (CC BY) license (https: / / creativecommons.org / licenses/by/4.0/).
Laboratory of Aquatic Biomedicine, College of Veterinary Medicine and Research Institute for Veterinary Science, Seoul National University, Seoul 08826, Korea; kjun1002@snu.ac.kr (J.K.); imagine5180@gmail.com (S.G.K.); hjoong1@nate.com (H.J.K.); giribiotek@gmail.com (S.S.G.); kasey.kim90@gmail.com (S.W.K.); 1sbin1129@naver.com (S.B.L.)

* Correspondence: parksec@snu.ac.kr; Tel.: +82-2-880-1282

\begin{abstract}
The increasing emergence of antimicrobial resistance has become a global issue. Therefore, many researchers have attempted to develop alternative antibiotics. One promising alternative is bacteriophage. In this study, we focused on a jumbo-phage infecting Salmonella isolated from exotic pet markets. Using a Salmonella strain isolated from reptiles as a host, we isolated and characterized the novel jumbo-bacteriophage pSal-SNUABM-04. This phage was investigated in terms of its morphology, host infectivity, growth and lysis kinetics, and genome. The phage was classified as Myoviridae based on its morphological traits and showed a comparatively wide host range. The lysis efficacy test showed that the phage can inhibit bacterial growth in the planktonic state. Genetic analysis revealed that the phage possesses a 239,626-base pair genome with 280 putative open reading frames, 76 of which have a predicted function and 195 of which have none. By genome comparison with other jumbo phages, the phage was designated as a novel member of Machinavirus composed of Erwnina phages.
\end{abstract}

Keywords: bacteriophage; jumbo-phage; reptile-associated salmonellosis; phage therapy; bacteriophage genome

\section{Introduction}

Bacteriophages are virus that can infect and lyse bacterial cells [1,2]. These bacterial viruses are considered promising alternatives to antibiotics because of their unique characteristics [1,2]. Phages have often a narrow range of host specificity [1,2], enabling their application to eradicate target bacteria without disrupting the normal flora [1,2]. Furthermore, bacteriophages show remarkable biosafety even with long-term administration [1,2]. These features make bacteriophages among the best alternatives to antibiotics [2].

Phages with genomes larger than $200 \mathrm{kbp}$ are known as jumbo-phages [3]. Because of their large genomes, jumbo-phages are highly diverse [3,4] and possess a larger number of genes in comparison to phages with shorter genomes [3]. These gene products may reduce the utilization of host proteins and make them less dependent on the host strains, making giant phages enable to have a wider host range $[3,5]$. However, even until recently many jumbo-phage gene products remained as hypothetical proteins. Although many jumbo-phage genomes remain uncharacterized, these bacterial viruses provide important clues for understanding bacteriophages in general [3-5].

In this study, we isolated and characterized a novel jumbo-bacteriophage, pSalSNUABM-04, which is virulent to Salmonella sp. We examined the biological and genomic features of this jumbo-phage and compared its genomic features to those of other jumbo-phages. 


\section{Materials and Methods}

\subsection{Bacterial Strains Used in This Study}

The Salmonella strain was isolated from pet reptiles. Samples were collected by swabbing the reptile skins and cloaca. Sixty skin swabs and 20 cloacal swabs were performed. After overnight enrichment in Rappaport-Vassiliadis R10 broth (Difco, Detroit, MI, USA) at $27^{\circ} \mathrm{C}$, the enrichment medium was spread on xylose lysine deoxycholate agar (Difco) and incubated overnight at $27^{\circ} \mathrm{C}$. Suspected colonies were sub-cultured by the streak plate method on fresh xylose lysine deoxycholate agar three times. The isolates were identified by $16 \mathrm{~S}$ rRNA gene sequencing performed by Macrogen (Seoul, Korea). Fourteen of the suspected isolates were confirmed to be Salmonella. The bacteria were stored at $-70{ }^{\circ} \mathrm{C}$ in tryptic soy broth (TSB; Difco) containing 15\% glycerol until use.

\subsection{Isolation and Characterization of the Phage pSal-SNUABM-04}

The S. enterica strain Sal-SNUABM-svn1, isolated from a Savannah monitor (Varanus exanthematicus) cloacal swab sample, was used to isolate the bacteriophage as described by Kim [1]. Briefly, environmental water samples collected in South Korea were filtered through $0.45 \mu \mathrm{m}$ membrane filters (Merck Millipore, Billerica, MA, USA). The filtered samples were mixed with TSB (1:1) into which an overnight cultured host strain $(1 \%, v / v)$ was inoculated. The suspension was incubated for $24 \mathrm{~h}$ at $27^{\circ} \mathrm{C}$. The presence of bacteriophage was verified by the conventional double-layer agar technique. Double-layered agar plates were incubated overnight at $27^{\circ} \mathrm{C}$. After observing plaque formation, single plaques were isolated with a Pasteur pipette 5 times. The isolated bacteriophage was designated as pSal-SNUABM-04.

The phage was characterized as described by Kim [1]. For phage adsorption analysis, the host bacterial strain in the exponential phase was infected with $10 \mu \mathrm{L}$ phage suspension (final multiplicity of infection, MOI, of 0.001 ), and the mixture was incubated at $27^{\circ} \mathrm{C}$. To titrate un-adsorbed phages, aliquots $(100 \mu \mathrm{L})$ were collected at $0,0.5,1,2,3,5,7,10,15$, 20 , and $30 \mathrm{~min}$ after phage infection. The aliquots were centrifuged at $12,000 \times \mathrm{g}$ for $3 \mathrm{~min}$, and the supernatants were diluted in $900 \mu \mathrm{L}$ phosphate-buffered saline and cultured by the conventional double-layered-agar method. A one-step growth curve was constructed, and the phage lysate was inoculated into the host bacterial strain culture in the exponential phase. The final MOI of the phage and bacterial cell mixture was 0.001. After more than $95 \%$ of the phages had adsorbed for 15 min according to the results of adsorption analysis, the mixtures were centrifuged at $12,000 \times g$ for $3 \mathrm{~min}$. The supernatant was discarded, and the remaining bacterial pellet was resuspended in preheated TSB and incubated at $27^{\circ} \mathrm{C}$ with shaking at $150 \mathrm{rpm}$. Aliquots $(100 \mu \mathrm{L})$ were collected every $10 \mathrm{~min}$ for $140 \mathrm{~min}$. Plaqueforming units were determined using the double-layered agar method. All experiments were performed in triplicate.

The host range was tested using the double-layered agar method. Bacterial strains isolated from reptiles in this study were used. The phage concentration used in this experiment was $1.0 \times 10^{6} \mathrm{PFU} / \mathrm{mL}$ and the experiments were performed in triplicate.

\subsection{Electron Microscopy of the Phage pSal-SNUABM-04}

Transmission electron microscopy (TEM) of pSal-SNUABM-04 was performed using a Talos L120C (FEI, Hillsboro, OR, USA). For TEM, phage concentration and purification were performed by polyethylene glycol $8000-\mathrm{NaCl}$ precipitation in $\mathrm{SM}$ buffer and the phage was stained with $2 \%$ uranyl acetate. The dimensions of individual phages were measured.

\subsection{In Vitro Planktonic Bacterial Lysis Assay}

A planktonic bacterial cell lysis assay was conducted to evaluate the lytic efficacy against the host strain. Under MOIs of $0.1,1$, and 10, we analyzed the turbid and clear lysis patterns of the phage. Overnight-cultured bacteria were inoculated into TSB to prepare a bacterial suspension of $10^{8}$ colony-forming units/mL. Next, $200 \mu \mathrm{L}$ of the bacterial suspension was dispensed into 96-well plates. The phage lysates were inoculated to reach 
the final MOIs mentioned above and the plates were cultured at $27^{\circ} \mathrm{C}$ with shaking at $150 \mathrm{rpm}$. The turbid and clear patterns of phage-bacteria suspensions were determined by measuring the $\mathrm{OD}_{600}$ with a VersaMax Microplate Reader (Molecular Devices, Sunnyvale, CA, USA). The measurement intervals were $30 \mathrm{~min}$ until $18 \mathrm{~h}$, and $60 \mathrm{~min}$ from 18 to $24 \mathrm{~h}$. After the assays, the mixtures were sub-cultured 3 times, and the phage sensitivity of the cultured bacteria was tested by the double-layered agar method. The experiments were performed in triplicate.

\subsection{Evaluation of Phage Stability under Different Thermal and $p H$ Conditions}

Phage stability was evaluated under different thermal and $\mathrm{pH}$ conditions. To test the $\mathrm{pH}$ stability of the phage, the phage lysate was inoculated in $1 \mathrm{~mL}$ of TSB prepared at different $\mathrm{pH}$ levels ( $\mathrm{pH} 2.0,4.0,5.0,7.0$, and 9.0) adjusted with $1 \mathrm{M} \mathrm{HCl}$ and $1 \mathrm{M} \mathrm{NaOH}$ solutions. The mixtures were incubated at $27^{\circ} \mathrm{C}$ for $2 \mathrm{~h}$, and phage titration was performed by dilution and conventional double-layered plating. The thermal stress stability of the phage was evaluated at different temperatures $\left(4^{\circ} \mathrm{C}, 20^{\circ} \mathrm{C}, 27^{\circ} \mathrm{C}\right.$, and $\left.37^{\circ} \mathrm{C}\right)$. After incubating the phage suspensions at different temperatures for $2 \mathrm{~h}$, titration was performed by dilution and plating. All analyses were performed with Sigmaplot 14.0 software (Systat Software Inc., IL, USA) using analysis of variance with Dunnett's post-hoc test. $P$ values $<0.05$ were considered significantly different.

\subsection{Phage Sequencing and Genome Analysis}

Phage DNA extraction was performed as described by Kim [1]. Purified phage genomic DNA was sequenced on an Illumina Hiseq2500 platform (San Diego, CA, USA) at Genotech (Daejeon, Korea). We used Unipro UGENE v35.0 to trim and assemble the reads. For putative open reading frame (ORF) prediction, Rapid Annotation using Subsystem Technology v2.0 (RAST), GeneMarkS v4.28, and protein BLAST were used [6,7]. tRNA detection was performed using tRNAscan-SE v2.0 [8]. The functions of the predicted ORFs were verified by BLAST searching. The conserved domains of the phage genome were searched by HHpred tool [9].

\subsection{Comparative Genome Analysis}

For phylogenetic analysis, the genome sequences of the jumbo phages were obtained from the GenBank database and aligned using Clustal W [10]. For single genome phylogeny analysis, we used sequences of the major capsid protein and terminase large subunit genes. Phylogenetic trees were constructed in MEGA v10.1.8 software using the maximum likelihood method with 1000 bootstrap replications [11]. For the whole-genome phylogenetic tree, we used the Genome-BLAST Distance Phylogeny method in the Virus Classification and Tree Building Online Resource (VICTOR) [12]. The resulting intergenomic distances (including 100 replicates each) were used to infer a balanced minimum evolution tree with branch support via FASTME including subtree pruning and regrafting postprocessing for the formula D0 [13]. The tree was visualized using FigTree [14]. Dot plots were drawn using Gepard at a word size of 10 [15]. The EZBiocloud ANI calculator was used to calculate the average nucleotide identity [16]. Comparative genome analysis of Machinavirus and pSal-SNUABM-04 was performed using Mauve [17].

\section{Results and discussions}

\subsection{Bacterial Strains Used in This Study}

Fourteen strains of Salmonella enterica bacteria were isolated from the swab samples. Among them, Sal-SNUABM-svn1 was used as the host strain (Table 1). The phage showed a wide host range towards reptile Salmonella $[71.42 \%(10 / 14)]$. The ability of the phage to infect a wide host range likely involves many genes encoding factors important in DNA replication and nucleotide metabolism, similar to other jumbo-phages examined in previous studies $[3,5]$. 
Table 1. Bacterial strains and bacterial susceptibility to pSal-SNUABM-04. - , plaque not formed; +, turbid plaque formation; ++ , clear plaque formation; +++ , more clear plaque formation.

\begin{tabular}{cccc}
\hline & \multicolumn{2}{c}{ Sampled Species } & $\begin{array}{c}\text { pSal-SNUABM- } \\
\text { 04 Susceptibility }\end{array}$ \\
\cline { 2 - 3 } Bacterial Strain & Common Name & Nomenclature & +++ \\
Sal-SNUABM-svn1 & Savannah monitor & Varanus exanthematicus & - \\
Sal-SNUABM-svn2 & Savannah monitor & Varanus exanthematicus & + \\
Sal-SNUABM-svn3 & Savannah monitor & Varanus exanthematicus & + \\
Sal-SNUABM-svn4 & Savannah monitor & Varanus exanthematicus & + \\
Sal-SNUABM-lp1 & Leopard gecko & Eublepharis macularius & - \\
Sal-SNUABM-bts1 & Common blue tongue skink & Tiliqua scincoides & ++ \\
Sal-SNUABM-mk1 & Milk snake & Lampropeltis triangulum & - \\
Sal-SNUABM-bks1 & Black king snake & Lampropeltis getula nigrita & - \\
Sal-SNUABM-gg1 & Tokay gecko & gecko gekko & + \\
Sal-SNUABM-bd1 & Bearded dragon & Pogona vitticeps & ++ \\
Sal-SNUABM-bd2 & Bearded dragon & Pogona vitticeps & + \\
Sal-SNUABM-bd3 & Bearded dragon & Pogona vitticeps & + \\
Sal-SNUABM-bs1 & Black rat snake & Pantherophis obsoletus & + \\
Sal-SNUABM-bp1 & Ball python & Python regius & + \\
\hline
\end{tabular}

\subsection{Biological Features of the Phage pSal-SNUABM-04}

Salmonella phages were isolated from water samples collected from the Nam-river, South Korea. Phage morphology was determined by TEM and classified based on the criteria proposed by Ackermann [18]. Phage pSal-SNUABM-04 was designated in the Myoviridae family and had an icosahedral head with a diameter of $80 \pm 3 \mathrm{~nm}$ and tail length of $116 \pm 10 \mathrm{~nm}$ (Figure 1A).

A
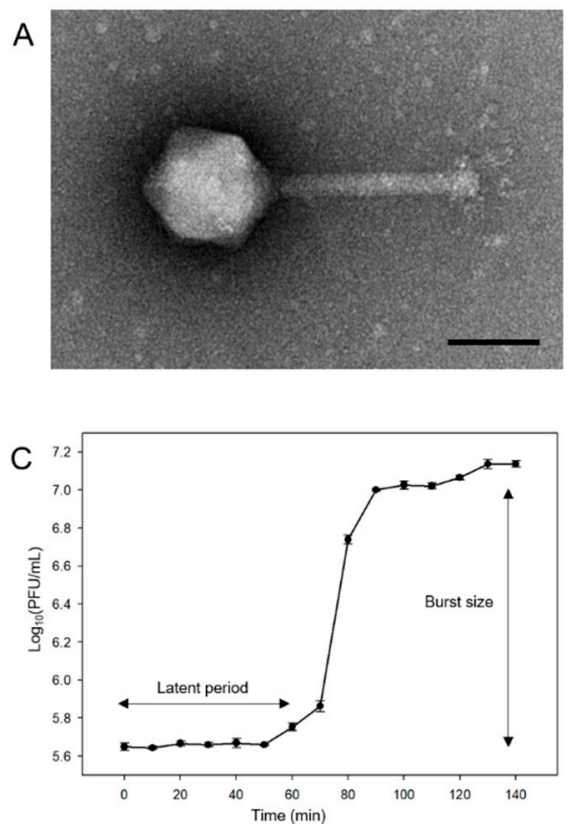
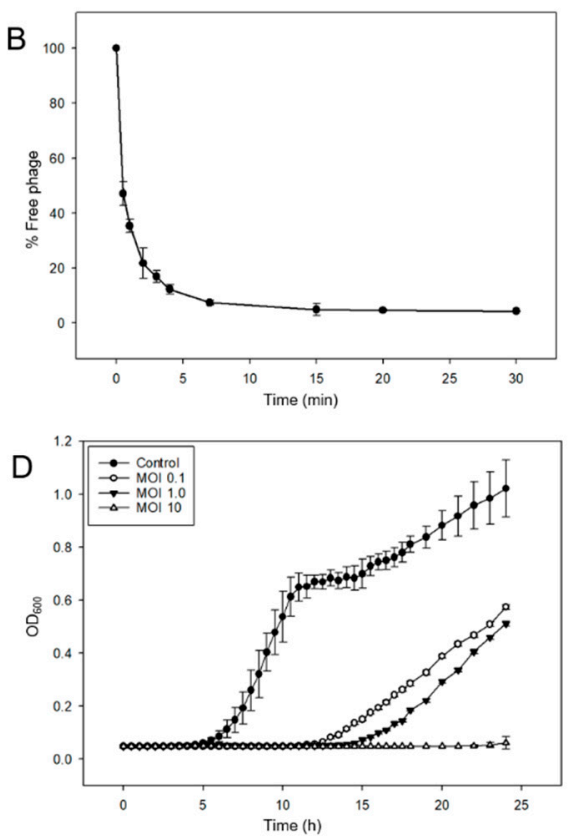

Figure 1. Morphological and biological features of phage pSal-SNUABM-04. (A) Transmission electron micrograph of pSal-SNUABM-04. Scale bar = $100 \mathrm{~nm}$. (B) Adsorption assay of pSal-SNUABM-04 to host strain Sal-SNUABM-svn1. (C) One-step growth curve of pSal-SNUABM-04 to host bacterial strain. (D) In vitro planktonic cell lysis efficacy assay of pSal-SNUABM-04 at MOI of 0.1, 1, and 10 against host bacterial strain.

The adsorption test showed that 95\% of the phage was adsorbed in 15 min (Figure 1B). The phage adsorption constant $\mathrm{k}$ was calculated as described in a previous study [19], 
$k=3.76 \times 10^{-10} \mathrm{~mL} / \mathrm{min}$. The latent period of phage pSal-SNUABM-04 was $60 \mathrm{~min}$ and the burst size was 29.11 (Figure 1C).

The results of the cell lysis assay against planktonic bacterial cells are shown in Figure 1D. In contrast to the continuous increase in the control group (non-phage-treated group), the phage-treated groups showed significant decreases in $\mathrm{OD}_{600}$ values. Gradual bacterial growth was observed in the 0.1 and 1 MOI groups. However, when the phage was treated at a high concentration $(\mathrm{MOI}=10)$, bacterial growth was inhibited. All cultured bacteria from the cell lysis assays showed sensitivity to phage pSal-SNUABM-04.

For plaque-forming unit determination, phage stability under different thermal and $\mathrm{pH}$ conditions was analyzed. At different $\mathrm{pH}$ levels, the phage titers decreased, particularly at lower $\mathrm{pH}$ levels of $\mathrm{pH} 4-5$, but not significantly at $\mathrm{pH} 5-9$ for $2 \mathrm{~h}$ (Figure 2A). In the thermal stability test, the phages were stable at $4{ }^{\circ} \mathrm{C}, 25^{\circ} \mathrm{C}$, and $27^{\circ} \mathrm{C}$, whereas stability was decreased at $37^{\circ} \mathrm{C}$ within $2 \mathrm{~h}$ (Figure 2B).
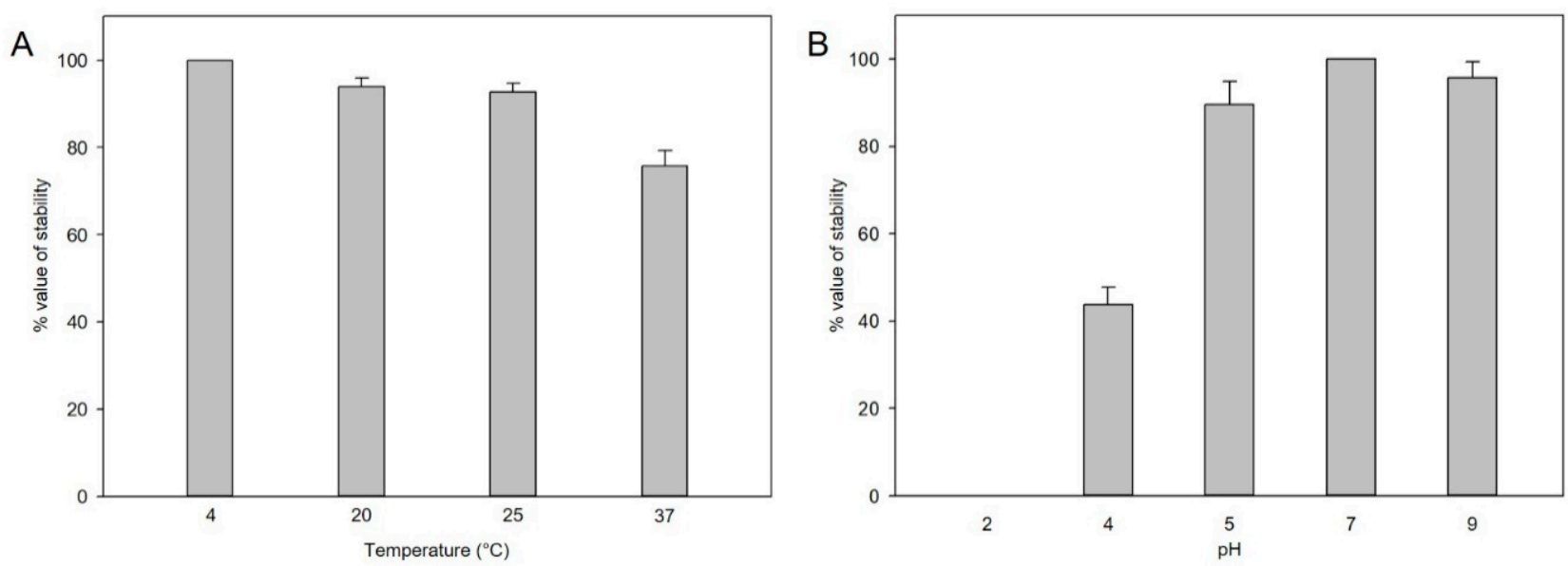

Figure 2. (A) Thermal stability of pSal-SNUABM-04. Phage lysates were incubated under different thermal conditions for $2 \mathrm{~h}$. (B) $\mathrm{pH}$ stability of pSal-SNUABM-04. Phage lysates were incubated under different $\mathrm{pH}$ conditions for $2 \mathrm{~h}$. Asterisks indicate statistically significant differences $(p<0.05), n=3$.

\subsection{General Features of the Phage pSal-SNUABM-04 Genome}

The whole genome of phage pSal-SNUABM-04 was sequenced and annotated (accession number; MT710307) (Figure 3). The phage genome consists of circular double-stranded DNA. The genome size was 239,626 base pairs with a GC content of $51.56 \%$. A total of 280 putative ORFs were predicted in the genome. Most putative genes (244 of 280; 87.14\%) were located on the positive strand and only 36 genes (36 of 280; $12.85 \%$ ) were on the negative strand. Of these, $76 \mathrm{ORFs}$ were determined to have a predicted function and 195 ORFs were classified as hypothetical proteins. The ORFs with a predicted function were classified into three categories; nucleotide metabolism-related, structure and packaging related, and lysis related genes. No genes were found to have predicted functions relating to virulence or lysogeny based on the currently available database. However, because most ORFs were predicted as hypothetical proteins, further investigation of the roles of the encoded gene products are needed. 


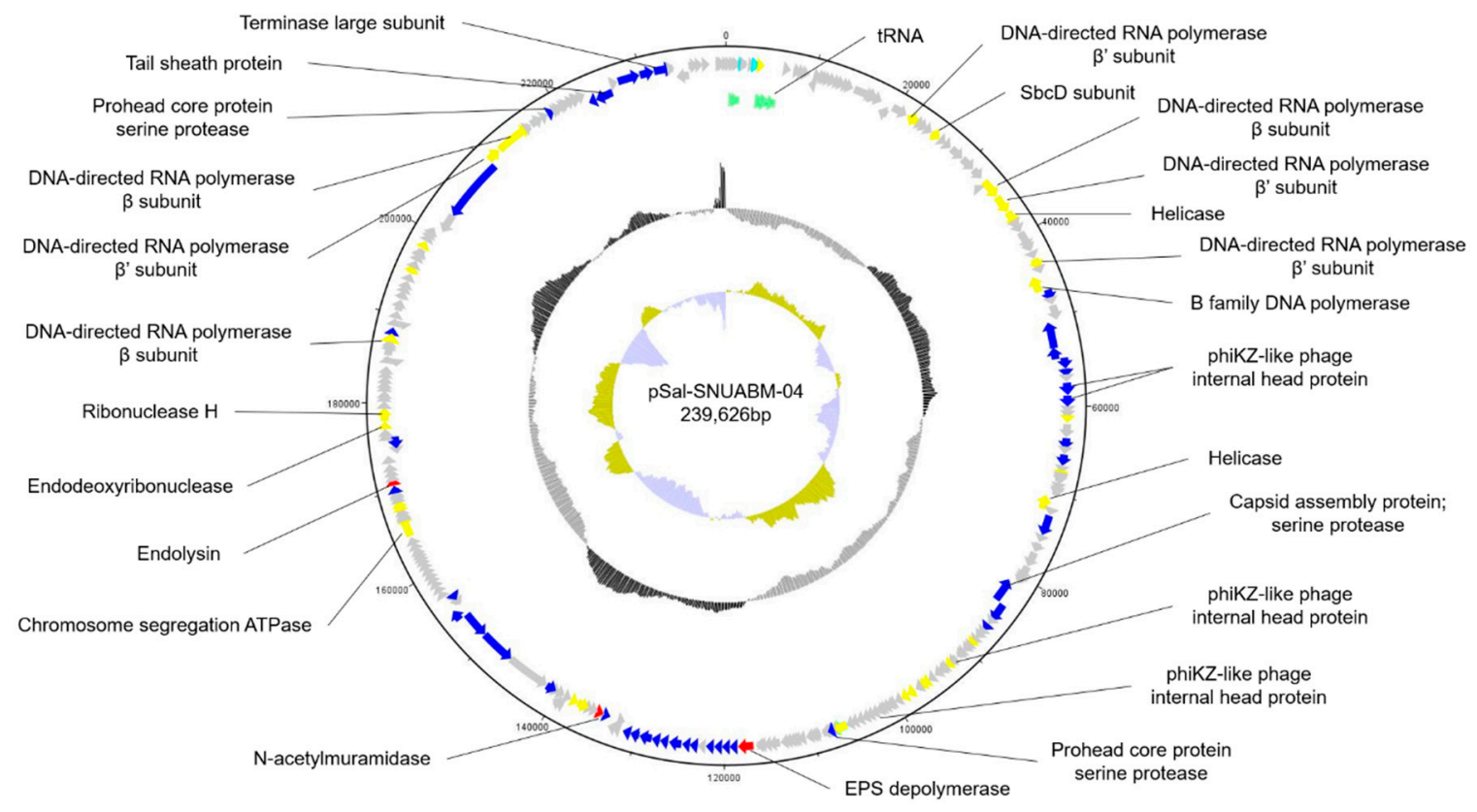

Figure 3. Genome map of pSal-SNUABM-04. The open reading frames (ORFs) are indicated by specific colors according to their functional categories. The GC skew is shown as inner circles holograms in cyan and green. GC content is indicated by black circular hologram. Red; lysis-related gene. Blue; structure and packaging related genes. Yellow; nucleotide metabolism-related genes. Gray; hypothetical gene. Green; tRNA.

In the phage genome, we found 10 putative tRNAs involved in phage genome transcription in the host. The presence of tRNA in the phage genome indicates phage adaptation to host bacteria. By compensating for phage codon usage, tRNA may contribute to phage infectivity and virulence.

Table S1 shows the general features of the predicted ORFs in the pSal-SNUABM-04 genome.

\subsection{Comparative Genomics of the pSal-SNUABM-04 Genome}

A BLAST search revealed that pSal-SNUABM-04 was highly related to the Machinavirus group ( $>96 \%$ similarity) composed of Erwinia-infecting jumbo-phages vB_EamM_Huxley, vB_EamM_Machina, vB_EamM_Parshik. To investigate and visualize the genomic distances between pSal-SNUABM-04 and other jumbo-phages, the phylogeny and dot plot method were performed with whole-genome and phage conserved gene sequences (major capsid protein, and terminase large subunit). The whole-genome phylogeny constructed by the VICTOR server and phylogeny-based dot plot revealed 12 clusters (Figure 4). The close-jumbo-phage cluster includes Erwinia phage vB_EamM_Huxley, vB_EamM_Machina, vB_EamM_Parshik, vB_EamM_ChrisDB, vB_EamM_Caitlin, Wellington, vB_EamM_Asessino, vB_EamM_Stratton, phiEaH2, Salmonella phage SPN3US, SPAsTU, Enterobacteria phage SEGD1, and Cronobacter phage CR5. The whole-genome phylogeny demonstrated not only the genome relationship of pSal-SNUABM-04 with Machinavirus, but the clue that the phage was separated recently from other cluster members. The dot plot supported the whole-genome phylogeny results, but also showed the large clustering of the group, exhibiting nucleotide similarity (blue square in Figure 4), composed of cluster 1 (vB_EamM_Huxley, vB_EamM_Machina, vB_EamM_Parshik, pSal-SNUABM-04), cluster 2 (vB_EamM_ChrisDB, vB_EamM_Caitlin), cluster 3 (vB_EamM_Asessino, vB_EamM_Stratton, phiEaH2, Salmonella phage SPN3US, SPAsTU, Enterobacteria phage SEGD1), and other non-clustered phages (Wellington and Cronobacter phage CR5). The phages in the big cluster also showed similar relationships in conserved gene sequence phylogeny. 

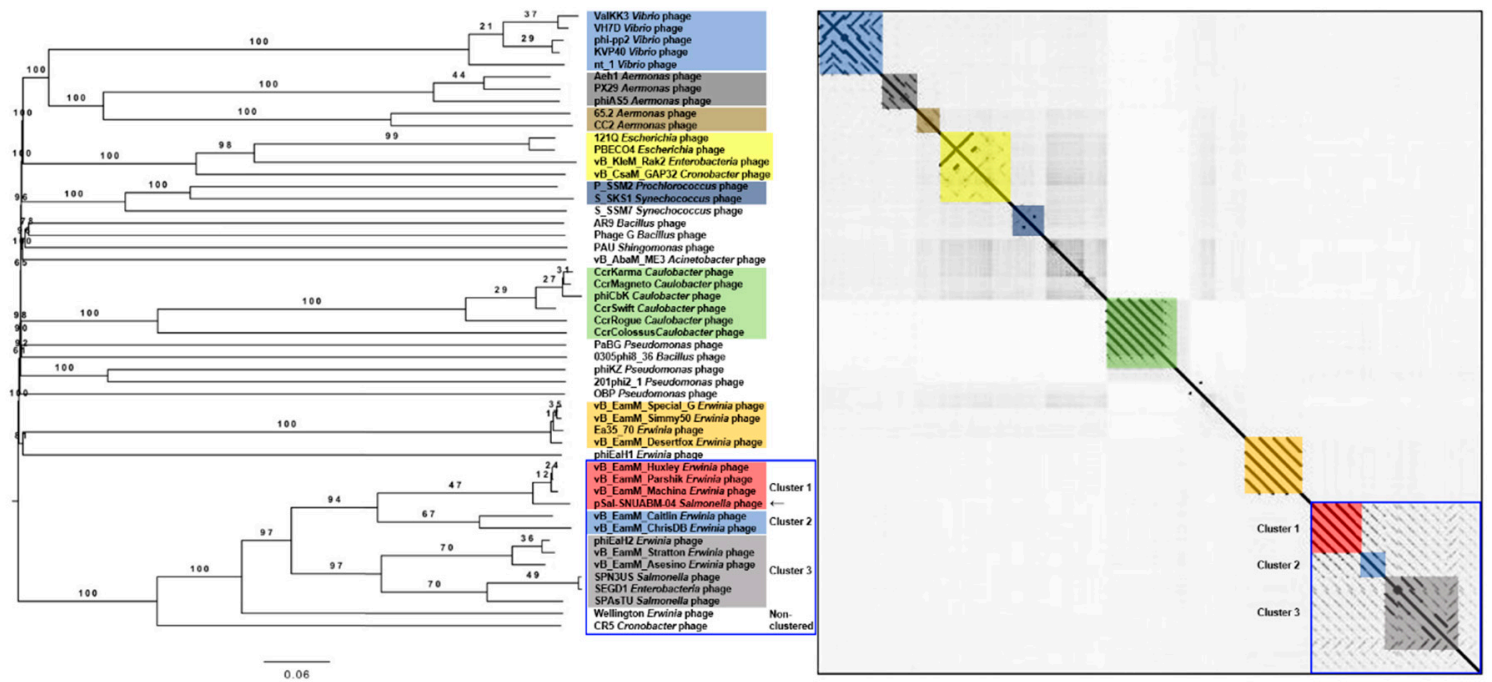

Figure 4. Comparative analysis of pSal-SNUABM-04 using whole-genome sequences. The phylogenetic tree was constructed using VICTOR with settings for prokaryotic viruses. The dot plot was generated with Gepard software at a word size of 10. Blue square; a large cluster composed of groups 1, 2,3, and two non-clustered phages.

We used two gene loci, major capsid protein (Figure 5) and terminase large subunit (Figure 6), to construct the phylogenetic tree and dot plot. Although there were a few differences in the three phylogenetic trees (whole genome, major capsid protein, and terminase large subunit), the cluster containing pSal-SNUABM-04 showed high relatedness. Both single-locus phylogenies exhibited high consistency with the whole genome phylogeny, indicating that pSal-SNUABM-04 can be assigned as a new species of Machinavirus.
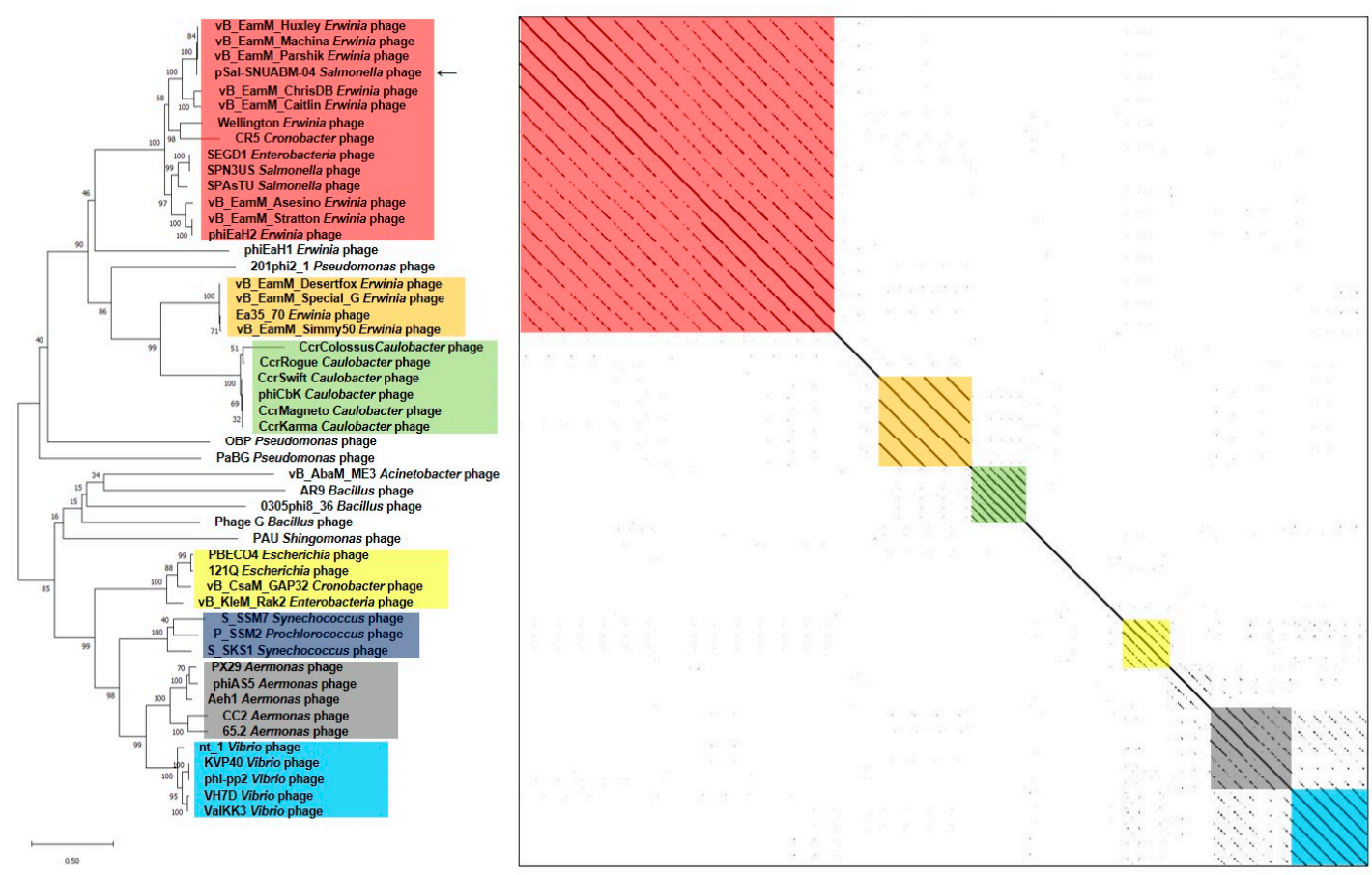

Figure 5. Comparative analysis of pSal-SNUABM-04 using major capsid protein sequences. The phylogenetic tree was constructed using MEGA-X by maximum likelihood with 1000 bootstrap replications. The dot plot was generated with Gepard software at a word size of 10 . 


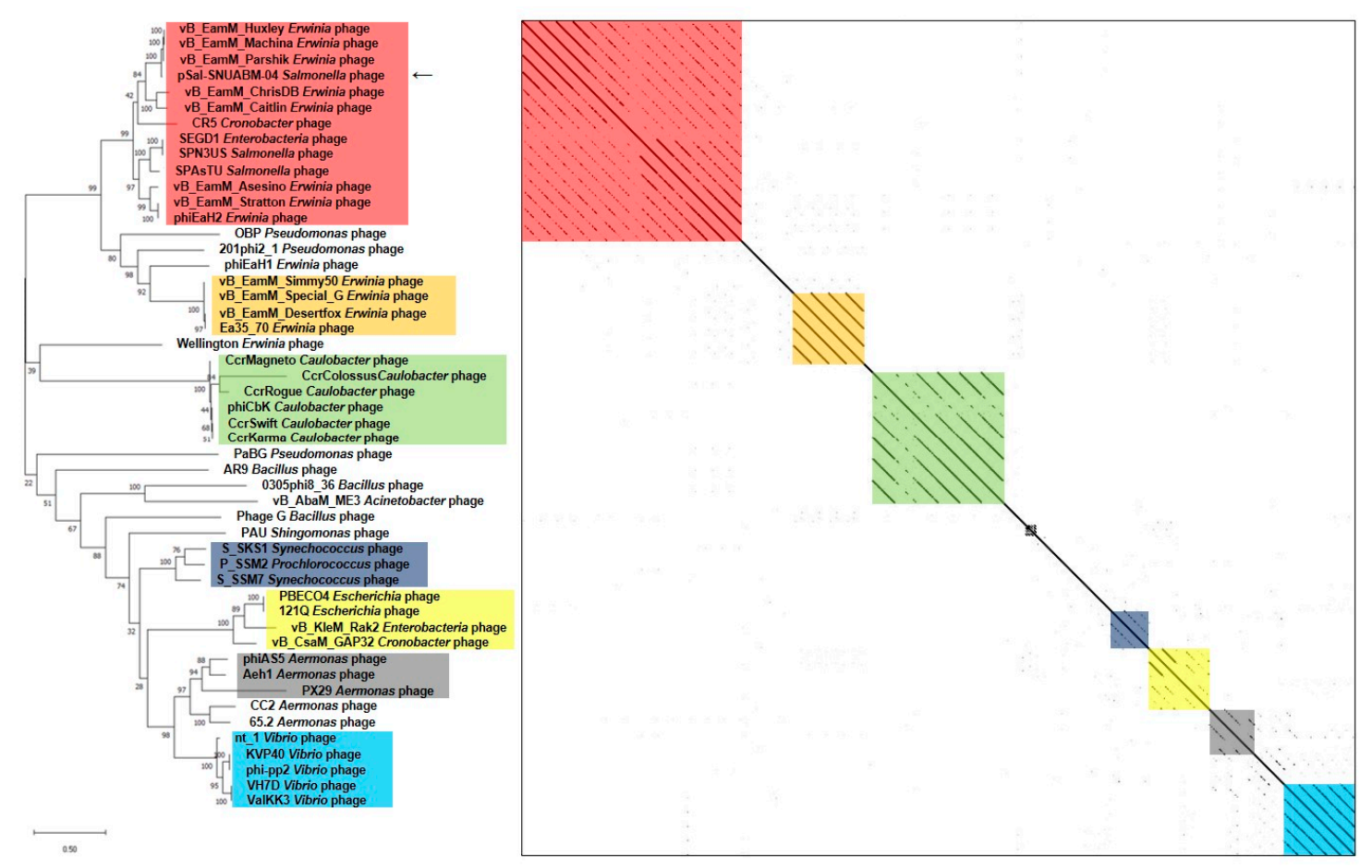

Figure 6. Comparative analysis of pSal-SNUABM-04 using terminase large subunit sequences. The phylogenetic tree was constructed using MEGA-X by maximum likelihood with 1000 bootstrap replications. The dot plot was generated with Gepard software at a word size of 10 .

Nucleotide homology was searched. The nucleotide and protein sequences of all ORFs were used for the BLAST search. The BLAST results showed a strong similarity between the ORFs of pSal-SNUABM-04,vB_EamM_Huxley, and other Machinavirus phages (Supplementary Table S1). The Ortho-average nucleotide identity (OrthoANI) values were calculated with whole-genome sequences. The calculated OrthoANI values of pSalSNUABM-04 and Machinavirus jumbo-phages were remarkably high (over 94.5\%), and the genomes of other close phages were $<70.42 \%$ and $>64.13 \%$ (Table S2). These results suggest that phage pSal-SNUABM-04 is closely associated with Machinavirus.

\subsection{Specific Features of the pSal-SNUABM-04 Genome}

\subsubsection{Nucleotide Metabolism-Related Genes}

RNA polymerase (RNAP) genes were recognized by BLASTn and domain search using HHpred tool. Seven ORFs were predicted as RNAPs, i.e., ORF 30, 41, 42, 49, 223, 244, and 245. The RNAPs were assigned as two large subunits $\beta$ and $\beta^{\prime}$, but no recognizable $\sigma$ factors were found.

The sequence similarity in phage pSal-SNUABM-04 RNAPs, and phage SPN3US RNAPs were searched in nucleotide BLAST. The three virion-associated RNAP subunit genes (vRNAP) of phage SPN3US showed high sequence similarity to three of pSalSNUABM-04 RNAP genes, i.e., ORF 49, 244, and 245 [20]. Non-virion-associated RNAPs (nvRNAP) were not aligned. The homologous vRNAP subunits were assigned as ORF 245 to the $\mathrm{v} \beta N$ subunit, ORF 244 to $v \beta^{\prime} \mathrm{N}$, and ORF 49 to $\mathrm{v} \beta^{\prime} \mathrm{M}$, whereas no $\mathrm{C}$-terminal gene homologs of the $\beta$ and $\beta^{\prime}$ subunits were detected. However, it is reasonable to consider it to be comprised of hypothetical gene proteins. The existence of vRNAPs genes has a big meaning in phage reproduction. Because the vRNAPs injected into host bacteria start gene transcription without host transcription factors, phage progeny production becomes more independent $[21,22]$.

The $\mathrm{SbcCD}$ is a complex frequently found in bacteria $[20,23,24]$. The complex has roles in ATP-dependent DNA repair and replication and is composed of two subunits, $\mathrm{SbcC}$ and 
$\mathrm{SbcD}$. SbcC, the larger subunit, is ATPase and the $\mathrm{SbcD}$ subunit shows double-stranded DNA exonuclease activity [20]. SPN3US phage and other giant phages also showed homologs of the SbcCD gene [20]. In the pSal-SNUABM-04 gene, the SbcD subunit, a Mre11 nuclease domain, was found in ORF 34, and the $\mathrm{SbcC}$ subunit, a chromosome segregation ATPase domain, was predicted at ORF 189 . The SbcCD complex is functionally grouped genes, but the homologs were placed in distance. This functional gene group splitting is a feature of phiKZ-related phage, suggesting unusual evolutional mechanisms [22].

\subsubsection{Structural and Packaging Related Genes}

In conserved domain search, phiKZ-like internal head protein was found in the ORF 60,61 , and 98 . The internal head structure, known as the inner body, is an additional substructure found in the capsids of some phiKZ-related giant phages [25]. The studies on this unusual cylindrical structure have reported that it is an essential feature of phiKZ-related phages [25-30]. However, the functions of the inner body have not been verified yet [25]. The inner body was hypothesized to have multi-function related to DNA packaging, packed genome structure, DNA ejection, or various roles in phage development [25,29].

The prohead serine protease genes, ORF 86,123 , and 249 , were predicted by sequencebased searches and protein homology detection. This prohead protease plays essential role in phage capsid morphogenesis [25,30].

\subsubsection{Lysis-Related Genes}

Of bacterial cell wall lysis genes, lytic glycosylase (ORF 200), extracellular polysaccharide (EPS) depolymerase, i.e., pectate lysate, (ORF 139) and N-acetylmuramidase (ORF 158), tail-associated lysozyme, i.e., fused to tape measure protein, (ORF 243) protein genes were identified. Phage-encoded enzymes attacking peptidoglycans were studied in many previous studies [31-38]. These enzymes have their distinct attack points which are linkage points between tetrapeptides and residues. EPS matrix secreted by bacteria makes phage hard to encounter with its targets. Therefore, these genes, especially ORF 139, verify the capacity of phage to lyse bacteria cells [36-38].

\subsubsection{Additional Function Genes}

The HslV protease, heat shock-induced locus gene product $\mathrm{V}$, was predicted at ORF 1 and 116 loci. The heat shock protein complex, HslVU, is an ATP-dependent protease that degrades selective proteins that are abnormal or heat-damaged $[39,40]$. The complex consists of two proteins, i.e., the protease subunit HslV and ATPase subunit HslU [40]. However, in the phage genome, only HslV subunit genes were encoded, whereas no HslU subunit was detected.

Bacterial ORFs encoding metal ion resistance were found in the phage genome. ORF 5 was found to be homologous to tellurite resistance protein, only terB domain of the ter operon (terZ-ABCDEF) [41]. Of the ter operons, ter B is an amphitropic peripheral membrane protein of bacterial cells [42]. A previous study suggested that terB protein may contribute to the reduction of tellurite [42].

\subsubsection{Horizontal Gene Transfer}

By metagenomic analysis with mauve system, genomes of Machinavirus and pSalSNUABM-04 were compared (Figure S1). These phages showed high similarity, i.e., approximately $94 \%$ OrthoANI, although pSal-SNUABM-04 phage was isolated by using Salmonella host. By sequence-based homolog searching, only approximately $7 \%(19 / 270)$ of pSal-SNUABM-04 ORFs were hits for genes of other giant phages as the closest homologs. Most of pSal-SNUABM-04 ORFs were highly related to Machinavirus, but seven ORFs (3, 70, $73,103,169,174$, and 178) showed very low similarity. In nucleotide and protein sequencebased searching, the homologs of ORFs were not identified except for ORF 70, which was predicted as a DNA ligase. The ORFs, which showed low similarity, underwent horizontal gene transfer [42-45]. Although it is not possible to verify where these genes were trans- 
ferred, it is reasonable that these transferred genes would make genomic and phenotypic differences in phage pSal-SNUABM-04 from the other Machinavirus members.

\section{Conclusions}

As antibiotic resistance has become a crucial issue worldwide, many studies have focused on alternatives to antibiotics [1,2]. Of the several alternatives, the bacteriophage is considered the most promising biocontrol agent owing to its many advantages such as the biosafety of in vivo phage administration and host bacteria-specific virulence [1,2]. Therefore, many studies on phages have been conducted globally [1,2,46-51]. A variety of phages have been isolated and characterized and their applications have been evaluated in many fields, including food safety, medicine, and aquacultures [46-51]. In addition, studies to overcome obstacles related to phage treatment were recently reported [52,53].

A few large phages with genomes greater than $200 \mathrm{kbp}$ have been identified [3-5]. These large phages, so-called jumbo-phages, contain several genes responsible for phage replication and their structures [3-5]. These functional genes in the jumbo-phage genome provide more host independence and wider host ranges compared to normal phage [3]. Therefore, studies of jumbo-phages may reveal additional information such as phage infection strategies, virion structure, and evolution [3].

In this study, we isolated a novel Salmonella phage, pSal-SNUABM-04, and characterized its biological and genomic properties. This phage showed vigorous lysis efficacy against Salmonella bacteria cells (Figure 1D). The ability of the phage to infect a wide host range likely involves many genes encoding factors important in DNA replication and nucleotide metabolism, similar to other jumbo-phages examined in previous studies $[1,4,5]$.

Genetic characterization revealed that pSal-SNUABM-04 is a potent new member of Machinavirus (Figures 4-6). Most phage pSal-SNUABM-04 genes were found to be homologous to Machinavirus genes. Additionally, the phylogenies and dot plots constructed from the whole genome, major capsid protein, and terminase large protein support the relationship between pSal-SNUABM-04 and Machinavirus members. Furthermore, the comparative analysis indicated horizontal gene transfers (Figure S1).

Supplementary Materials: The following are available online at https:/ /www.mdpi.com/1999-491 5/13/1/27/s1, Figure S1: Whole genome comparison of phages in Machinavirus cluster, Table S1: Functional categories and homologies of the predicted open reading frames (ORFs) in pSal-SNUABM04, Table S2: Calculations of average nucleotide identity (ANI) between pSal-SNUABM-04 and other jumbo-phages.

Author Contributions: Conceptualization, J.K. and S.C.P.; methodology, J.K. and S.S.G.; investigation, H.J.K.; writing—original draft preparation, J.K.; review, S.G.K., S.B.L., and S.W.K.; editing, S.C.P.; project administration, J.K.; funding acquisition, S.C.P. All authors have read and agreed to the published version of the manuscript.

Funding: This research was supported by the Cooperative Research Program of the Center for Companion Animal Research (PJ013985032020) of the Rural Development Administration, Republic of Korea and the Basic Science Research Program through the National Research Foundation of Korea (NRF), funded by the Ministry of Education (2018R1D1A1A02086128).

Institutional Review Board Statement: Not applicable.

Informed Consent Statement: Not applicable.

Data Availability Statement: The data presented in this study are available in supplementary material here.

Conflicts of Interest: The authors declare no conflict of interest.

\section{References}

1. Kim, S.G.; Jun, J.W.; Giri, S.S.; Yun, S.; Kim, H.J.; Kang, J.W.; Han, S.J.; Jeong, D.; Park, S.C. Isolation and characterisation of pVa-21, a giant bacteriophage with anti-biofilm potential against Vibrio alginolyticus. Sci. Rep. 2019, 9, 1-10. [CrossRef]

2. Kutateladze, M.; Adamia, R. Bacteriophages as potential new therapeutics to replace or supplement antibiotics. Trends Biotechnol. 2010, 28, 591-595. [CrossRef] 
3. Yuan, Y.; Gao, M. Jumbo bacteriophages: An overview. Front. Microbiol. 2017, 8, 403. [CrossRef] [PubMed]

4. Sharma, R.; Pielstick, B.A.; Bell, K.A.; Nieman, T.B.; Stubbs, O.A.; Yeates, E.L.; Baltrus, D.A.; Grose, J.H. A Novel, Highly Related Jumbo Family of Bacteriophages That Were Isolated Against Erwinia. Front. Microbiol. 2019, 10, 1533. [CrossRef] [PubMed]

5. Olszak, T.; Danis-Wlodarczyk, K.; Arabski, M.; Guła, G.; Maciejewska, B.; Wasik, S.; Lood, C.; Higgins, G.; Harvey, B.J.; Lavigne, R.; et al. Pseudomonas aeruginosa PA5oct Jumbo Phage Impacts Planktonic and Biofilm Population and Reduces Its Host Virulence. Viruses 2019, 11, 1089. [CrossRef] [PubMed]

6. Aziz, R.K.; Bartels, D.; Best, A.A.; DeJongh, M.; Disz, T.; Edwards, R.A.; Formsma, K.; Gerdes, S.; Glass, E.M.; Kubal, M.; et al. The RAST Server: Rapid Annotations using Subsystems Technology. BMC Genom. 2008, 9, 1-15. [CrossRef]

7. Besemer, J.; Lomsadze, A.; Borodovsky, M. GeneMarkS: A self-training method for prediction of gene starts in microbial genomes. Implications for finding sequence motifs in regulatory regions. Nucleic Acids Res. 2001, 29, 2607-2618. [CrossRef]

8. Lowe, T.M.; Chan, P.P. tRNAscan-SE On-line: Integrating search and context for analysis of transfer RNA genes. Nucleic Acids Res. 2016, 44, W54-W57. [CrossRef]

9. Zimmermann, L.; Stephens, A.; Nam, S.-Z.; Rau, D.; Kübler, J.; Lozajic, M.; Gabler, F.; Söding, J.; Lupas, A.N.; Alva, V. A Completely Reimplemented MPI Bioinformatics Toolkit with a New HHpred Server at its Core. J. Mol. Biol. 2018, 430, 2237-2243. [CrossRef]

10. Thompson, J.D.; Higgins, D.G.; Gibson, T.J. CLUSTAL W: Improving the sensitivity of progressive multiple sequence alignment through sequence weighting, position-specific gap penalties and weight matrix choice. Nucleic Acids Res. 1994, 22, 4673-4680. [CrossRef]

11. Kumar, S.; Stecher, G.; Li, M.; Knyaz, C.; Tamura, K. MEGA X: Molecular Evolutionary Genetics Analysis across Computing Platforms. Mol. Biol. Evol. 2018, 35, 1547-1549. [CrossRef]

12. Meier-Kolthoff, J.P.; Göker, M. VICTOR: Genome-based phylogeny and classification of prokaryotic viruses. Bioinformatics 2017, 33, 3396-3404. [CrossRef] [PubMed]

13. Farris, J.S. Estimating Phylogenetic Trees from Distance Matrices. Am. Nat. 1972, 106, 645-668. [CrossRef]

14. FigTree 1.4.3-A graphical viewer of phylogenetic trees and a program for producing publication-ready figures. Available online: http:/ / tree.bio.ed.ac.uk/software/figtree/ (accessed on 11 November 2020).

15. Krumsiek, J.; Arnold, R.; Rattei, T. Gepard: A rapid and sensitive tool for creating dot plots on genome scale. Bioinformatics 2007, 23, 1026-1028. [CrossRef] [PubMed]

16. Yoon, S.-H.; Ha, S.-M.; Lim, J.; Kwon, S.; Chun, J. A large-scale evaluation of algorithms to calculate average nucleotide identity. Antonie van Leeuwenhoek 2017, 110, 1281-1286. [CrossRef]

17. Darling, A.E.; Mau, B.; Blattner, F.R.; Perna, N.T. Mauve: Multiple Alignment of Conserved Genomic Sequence with Rearrangements. Genome Res. 2004, 14, 1394-1403. [CrossRef]

18. Ackermann, H.-W. 5500 Phages examined in the electron microscope. Arch. Virol. 2006, 152, 227-243. [CrossRef]

19. Hyman, P.; Abedon, S.T. Practical Methods for Determining Phage Growth Parameters. In Bioinformatics in MicroRNA Research; Springer Science and Business Media LLC: New York, NY, USA, 2009; Volume 501, pp. 175-202.

20. Thomas, J.A.; Quintana, A.D.B.; Bosch, M.A.; De Peña, A.C.; Aguilera, E.; Coulibaly, A.; Wu, W.; Osier, M.V.; Hudson, A.O.; Weintraub, S.T.; et al. Identification of Essential Genes in the Salmonella Phage SPN3US Reveals Novel Insights into Giant Phage Head Structure and Assembly. J. Virol. 2016, 90, 10284-10298. [CrossRef]

21. Lewis, R.; Clooney, A.G.; Stockdale, S.R.; Buttimer, C.; Draper, L.A.; Ross, R.P.; Hill, C. Isolation of a Novel Jumbo Bacteriophage Effective Against Klebsiella aerogenes. Front. Med. 2020, 7, 67. [CrossRef]

22. Ceyssens, P.J.; Minakhin, L.; Van den Bossche, A.; Yakunina, M.; Klimuk, E.; Blasdel, B.; De Smet, J.; Noben, J.P.; Bläsi, U.; Severinov, K.; et al. Development of giant bacteriophage $\phi K Z$ is independent of the host transcription apparatus. J. Virol. 2014, 88, 10501-10510. [CrossRef]

23. Miller, E.S.; Kutter, E.; Mosig, G.; Arisaka, F.; Kunisawa, T.; Rüger, W. Bacteriophage T4 Genome. Microbiol. Mol. Biol. Rev. 2003, 67, 86-156. [CrossRef] [PubMed]

24. Storvik, K.A.M.; Foster, P.L. The SMC-Like Protein Complex SbcCD Enhances DNA Polymerase IV-Dependent Spontaneous Mutation in Escherichia coli. J. Bacteriol. 2010, 193, 660-669. [CrossRef] [PubMed]

25. Thomas, J.A.; Weintraub, S.T.; Wu, W.; Winkler, D.C.; Cheng, N.; Steven, A.C.; Black, L.W. Extensive proteolysis of head and inner body proteins by a morphogenetic protease in the giant Pseudomonas aeruginosa phage $\varphi$ KZ. Mol. Microbiol. 2012, 84, 324-339. [CrossRef] [PubMed]

26. Wu, W.; Thomas, J.A.; Cheng, N.; Black, L.W.; Steven, A.C. Bubblegrams reveal the inner body structure of $\phi K Z$. Science 2012, 335, 10-1126. [CrossRef] [PubMed]

27. Black, L.W.; Thomas, J.A. Condensed Genome Structure. In Advances in Experimental Medicine and Biology; Springer Science and Business Media LLC: New York, NY, USA, 2011; Volume 726, pp. 469-487.

28. Krylov, V.N.; Smirnova, T.A.; Minenkova, I.B.; Plotnikova, T.G.; Zhazikov, I.Z.; Khrenova, E.A. Pseudomonas bacteriophage contains an inner body in its capsid. Can. J. Microbiol. 1984, 30, 758-762. [CrossRef] [PubMed]

29. Ali, B.; Desmond, M.I.; Mallory, S.A.; Benítez, A.D.; Buckley, L.J.; Weintraub, S.T.; Osier, M.V.; Black, L.W.; Thomas, J.A. To Be or Not to Be T4: Evidence of a Complex Evolutionary Pathway of Head Structure and Assembly in Giant Salmonella Virus SPN3US. Front. Microbiol. 2017, 8, 2251. [CrossRef]

30. Thomas, J.A.; Black, L.W. Mutational analysis of the Pseudomonas aeruginosa myovirus $\phi K Z$ morphogenetic protease gp175. J. Virol. 2013, 87, 8713-8725. [CrossRef]

31. Young, R. Bacteriophage lysis: Mechanism and regulation. Microbiol. Mol. Biol. Rev. 1992, 56, 430-481. [CrossRef] 
32. Schmelcher, M.; Donovan, D.M.; Loessner, M.J. Bacteriophage endolysins as novel antimicrobials. Futur. Microbiol. 2012, 7, 1147-1171. [CrossRef]

33. Matamp, N.; Bhat, S.G. Phage Endolysins as Potential Antimicrobials against Multidrug Resistant Vibrio alginolyticus and Vibrio parahaemolyticus: Current Status of Research and Challenges Ahead. Microorganisms 2019, 7, 84. [CrossRef]

34. Tišáková, L.; Godány, A. Bacteriophage endolysins and their use in biotechnological processes. J. Microbiol. Biotechnol. Food Sci. 2020, 9, 164-170.

35. Latka, A.; Maciejewska, B.; Majkowska-Skrobek, G.; Briers, Y.; Drulis-Kawa, Z. Bacteriophage-encoded virion-associated enzymes to overcome the carbohydrate barriers during the infection process. Appl. Microbiol. Biotechnol. 2017, 101, 3103-3119. [CrossRef] [PubMed]

36. Gutiérrez, D.; Briers, Y.; Rodríguez-Rubio, L.; Martínez, B.; Rodríguez, A.; Lavigne, R.; García, P. Role of the pre-neck appendage protein (Dpo7) from phage vB_SepiS-phiIPLA7 as an anti-biofilm agent in Staphylococcal species. Front. Microbiol. 2015, 6, 1315. [CrossRef] [PubMed]

37. Sutherland, I.W.; Hughes, K.A.; Skillman, L.C.; Tait, K. The interaction of phage and biofilms. FEMS Microbiol. Lett. 2004, 232, 1-6. [CrossRef]

38. Knecht, L.E.; Veljkovic, M.; Fieseler, L. Diversity and Function of Phage Encoded Depolymerases. Front. Microbiol. 2020, 10, 2949. [CrossRef]

39. Sousa, M.C.; Trame, C.B.; Tsuruta, H.; Wilbanks, S.M.; Reddy, V.S.; McKay, D.B. Crystal and Solution Structures of an HslUV Protease-Chaperone Complex. Cell 2000, 103, 633-643. [CrossRef]

40. Rohrwild, M.; Coux, O.; Huang, H.C.; Moerschell, R.P.; Yoo, S.J.; Seol, J.H.; Chung, C.H.; Goldberg, A.L. HslV-HslU: A novel ATPdependent protease complex in Escherichia coli related to the eukaryotic proteasome. Proc. Natl. Acad. Sci. USA 1996, 93, 5808-5813. [CrossRef]

41. Taylor, D.E. Bacterial tellurite resistance. Trends Microbiol. 1999, 7, 111-115. [CrossRef]

42. Hendrix, R.W. Bacteriophages: Evolution of the Majority. Theor. Popul. Biol. 2002, 61, 471-480. [CrossRef]

43. Haggård-Ljungquist, E.; Halling, C.; Calendar, R. DNA sequences of the tail fiber genes of bacteriophage P2: Evidence for horizontal transfer of tail fiber genes among unrelated bacteriophages. J. Bacteriol. 1992, 174, 1462-1477. [CrossRef]

44. Rokyta, D.R.; Burch, C.L.; Caudle, S.B.; Wichman, H.A. Horizontal Gene Transfer and the Evolution of Microvirid Coliphage Genomes. J. Bacteriol. 2006, 188, 1134-1142. [CrossRef] [PubMed]

45. McCarthy, A.; Witney, A.A.; Lindsay, J. Staphylococcus aureus Temperate Bacteriophage: Carriage and Horizontal Gene Transfer is Lineage Associated. Front. Cell. Infect. Microbiol. 2012, 2, 6. [CrossRef] [PubMed]

46. Kakasis, A.; Panitsa, G. Bacteriophage therapy as an alternative treatment for human infections. A comprehensive review. Int. J. Antimicrob. Agents 2019, 53, 16-21. [CrossRef] [PubMed]

47. Sulakvelidze, A.; Alavidze, Z.; Morris, J.G. Bacteriophage Therapy. Antimicrob. Agents Chemother. 2001, 45, 649-659. [CrossRef]

48. Sulakvelidze, A.; Kutter, E. Bacteriophage Therapy in Humans. In Bacteriophages; CRC Press: Washington, DC, USA, $2004 ;$ pp. 381-426.

49. Eaton, M.D.; Bayne-Jones, S. Bacteriophage therapy: Review of the principles and results of the use of bacteriophage in the treatment of infections. JAMA 1934, 103, 1769-1776. [CrossRef]

50. Berghman, L.R.; Abi-Ghanem, D.; Waghela, S.D.; Ricke, S.C. Antibodies: an alternative for antibiotics? Poult. Sci. 2005, 84, 660-666. [CrossRef]

51. Mingmongkolchai, S.; Panbangred, W. Bacillus probiotics: an alternative to antibiotics for livestock production. J. Appl. Microbiol. 2018, 124, 1334-1346. [CrossRef]

52. Kim, S.G.; Giri, S.S.; Yun, S.; Kim, H.J.; Kim, S.W.; Kang, J.W.; Han, S.J.; Kwon, J.; Oh, O.T.; Jun, J.W.; et al. Synergistic Phagesurfactant Combination Clears IgE-promoted Staphylococcus aureus Aggregation in Vitro and Enhances the Effect in Vivo. J. Antimicrob. Agents 2020, 105997. [CrossRef]

53. Singla, S.; Harjai, K.; Katare, O.P.; Chhibber, S. Encapsulation of Bacteriophage in Liposome Accentuates Its Entry in to Macrophage and Shields It from Neutralizing Antibodies. PLoS ONE 2016, 11, e0153777. [CrossRef] 